\title{
Epidemiology and antibiogram of Riemerella anatipestifer isolated from waterfowl slaughterhouses in Taiwan
}

\author{
Fei-Fei Chang ${ }^{1}$, Chang-Chieh Chen ${ }^{2}$, \\ Shao-Hung Wang ${ }^{3}$, Chiou-Lin Chen ${ }^{1}$ \\ ${ }^{1}$ Department of Veterinary Medicine, National Chiayi University, 60054 Chiayi, Taiwan, Republic of China \\ ${ }^{2}$ Green Energy and Environment Research Laboratories, Industrial Technology Research Institute, \\ 31057 Hsinchu, Taiwan, Republic of China \\ ${ }^{3}$ Department of Microbiology, Immunology, and Biopharmaceuticals, National Chiayi University, \\ 60004 Chiayi, Taiwan, Republic of China \\ shwang@mail.ncyu.edu.tw; chulin@mail.ncyu.edu.tw
}

Received: July 27, 2018 Accepted: January 11, 2019

\begin{abstract}
Introduction: Laryngeal swab samples collected from three waterfowl slaughterhouses in central Taiwan were cultured and suspected isolates of Riemerella anatipestifer were identified by API 20NE and 16S rDNA PCR. Material and Methods: Serum agglutination was used for serotyping, and antimicrobial susceptibility was tested. Results: Seventy-six $R$. anatipestifer isolates were detected, and the prevalences in the ducks and geese were $12.3 \%(46 / 375)$ and $8.0 \%(30 / 375)$, respectively. The positive isolation rates were $65.6 \%$ for all arriving waterfowl, $76.0 \%$ for birds in the holding area, $1.6 \%$ for defeathered carcasses, but zero for degummed carcasses. A PCR examination detected $R$. anatipestifer in the slaughtering area frequently. Serotype B was dominant in both duck (34.8\%) and goose (46.7\%) isolates, but the wide serotype distribution may very well impede vaccination development. All isolates were resistant to colistin, and $79.7 \%$ were resistant to more than three common antibiotics. Conclusion: The results proved that most ducks had encountered antibiotic-resistant $R$. anatipestifer in rearing, which suggests that the bacterium circulates in asymptomatic waterfowl. It is worth noting that most waterfowl farms were found to harbour $R$. anatipestifer, and contaminated slaughterhouses are a major risk factor in its spread. Effective prevention and containment measures should be established there to interrupt the transmission chain of $R$. anatipestifer.
\end{abstract}

Keywords: waterfowl, Riemerella anatipestifer, slaughterhouse, contamination, antibiotic resistance, Taiwan.

\section{Introduction}

Riemerella anatipestifer causes infections in waterfowl and has an adverse economic effect on waterfowl husbandry. It is a rod-shaped, Gram-negative bacterium infection with which results in $20 \%-30 \%$ morbidity and 5\%-20\% mortality in waterfowl displaying septicaemia and infectious serositis $(12,16$, 17). Respiratory tract and skin wounds are speculated to play important roles in $R$. anatipestifer transmission (29). Poor sanitation, nutrient deficiency, adverse (e.g. stressful) environments or climates, and concomitant diseases usually predispose fowl to outbreaks of $R$. anatipestifer infection, especially those under 8 weeks old $(3,26,34)$. In a survey of samples of waterfowl in Taiwan between 2006 and 2007, the significant prevalence $(84.4 \%)$ of $R$. anatipestifer in farms revealed the potential risk of an outbreak $(4,14)$. $R$. anatipestifer infection in young ducklings results in high morbidity (75\%), but older adults usually suffer from chronic subclinical diseases or are even asymptomatic (11).

High genetic diversity and serotype variation was reported among field $R$. anatipestifer isolates, with more than 21 serotypes found $(1,22,25,29,31)$. Although several kinds of vaccines against $R$. anatipestifer have been developed, such as attenuated, inactivated, and subcellular variants (7, 15, $20,24,28)$, high antigenic diversity and low crossprotection impede their application in the field. Antimicrobial agents and improved biosecurity are currently applied to prevent and control $R$. anatipestifer 
infection in waterfowl farming; however, the increasing resistance to common antibiotics in $R$. anatipestifer seriously challenges the treatment (5).

Epidemic studies of zoonotic Salmonella and Campylobacter in poultry slaughterhouses have been carried out worldwide $(23,32)$. However, relatively little epidemiological research on $R$. anatipestifer has been undertaken in the field, possibly due to its narrow host range and the scarcity of clinical symptoms in adult birds. In this study, an epidemiological investigation of $R$. anatipestifer in slaughterhouses was conducted, including testing for it in the birds on arrival, the carcasses being processed, the machines, and the environments.

\section{Material and Methods}

Sampling methods. First, to evaluate the prevalence of $R$. anatipestifer in waterfowl, laryngeal and conjunctival swab samples (2) were collected from three slaughterhouses in central Taiwan housing and processing ducks and geese that were transported from farms in central and southern Taiwan. Cochran's formula $\left(\mathrm{n}=\mathrm{Z}^{2} \mathrm{PQ} / \mathrm{e}^{2}\right.$, where $\mathrm{n}=$ sample size, $\mathrm{Z}=$ the desired confidence levels, $\mathrm{P}=$ expected prevalence, $\mathrm{Q}=1-\mathrm{P}$, and $\mathrm{e}=$ the desired level of precision (8)) was used to calculate a representative sample size in this study. Thereafter, brain heart infusion agar was used for the bacterial cultures of 125 ducks ( 25 birds per flock) at various stages of the slaughtering process, including laryngeal and conjunctival swabs of the birds on arriving and before slaughter, and wash solutions of defeathered or degummed carcasses (sampled by a oneminute shaking with $150 \mathrm{~mL}$ of sterile normal saline, followed by bacterial isolation from $15 \mathrm{~mL}$ of this solution). The environmental samples survey was performed in two slaughterhouses, where the workspace and machines coming into contact with the birds are disinfected with hot water and sodium hypochlorite after each processing. Before the disinfection, five samples for each of the 16 selected sites were conducted at different sampling spots: (1) the samples of pool water, bottom mud, faeces, drinking water, and fodder in holding areas, (2) surface swabs of the workbench top, defeathering machine, scalding machine, and transport machines, and (3) samples of the waste feathers, water supply, and wastewater from the waterfowl scalding, defeathering, degumming, organ removal, purge, and slaughtering areas.

Isolation of $\boldsymbol{R}$. anatipestifer. Isolation of $R$. anatipestifer from swab samples was performed by the streak method with blood agar plates (Trypticase soy agar with $5 \%$ sheep blood). Solution samples were pelleted at $3,000 \times \mathrm{g}$ for $10 \mathrm{~min}$, vortexed for $15 \mathrm{~s}$ with $10 \mathrm{~mL}$ of sterile normal saline, spun again to remove supernatants, and then streaked on blood agar plates. The agar plates were first cultivated at $37^{\circ} \mathrm{C}$ supplemented with $5 \% \mathrm{CO}_{2}$ for $24 \mathrm{~h}$, then the plates and MacConkey agar were used to cultivate the suspicious bacterial colonies for morphological identification and selection tests. All isolates were identified by a commercial API 20NE (bioMérieux, France).

Molecular identification of $\boldsymbol{R}$. anatipestifer. To prepare the DNA, a bacterial colony was picked up and suspended in $500 \mu \mathrm{L}$ of TAE buffer, which was then boiled for $10 \mathrm{~min}$ and centrifuged at $16,000 \times \mathrm{g}$ for $1 \mathrm{~min}$. The supernatant was directly used as the DNA template for the PCR. A 16S rDNA amplification PCR method previously described (18) used to molecularly identify $R$. anatipestifer isolates. Reactions containing an RA-5' (TCG AGA TTT GCA TCA CTT CGC ATT G) primer paired with an RA-3' (GCT AGT CTT GAG TAT AGT TGA GCT AGC) primer were performed on a MyCycler thermal cycler (Bio-Rad, USA) with the following amplification settings: initial denaturation at $94^{\circ} \mathrm{C}$ for $5 \mathrm{~min}, 35$ cycles of amplification (denaturation at $94^{\circ} \mathrm{C}$ for $5 \mathrm{~s}$, annealing at $50^{\circ} \mathrm{C}$ for $50 \mathrm{~s}$, and extension at $72^{\circ} \mathrm{C}$ for $50 \mathrm{~s}$ ), and a final extension at $72^{\circ} \mathrm{C}$ for $10 \mathrm{~min}$. The amplified products (645 bp) were electrophoresed on $2 \%$ agarose gels, stained with ethidium bromide, and then recorded and analysed using a GeneFlash system (Syngene, UK).

Serotyping of $\boldsymbol{R}$. anatipestifer. R. anatipestifer isolates were serotyped by the Animal Health Research Institute (AHRI), Taiwan, using the slide agglutination method. In short, $20 \mu \mathrm{L}$ of standard antiserum for $R$. anatipestifer typing was mixed with the same volume of single colony suspension from each isolate. A positive reaction was indicated by clumping of bacteria within 1 to $2 \mathrm{~min}$.

Antibiotic susceptibility assay. A disc diffusion assay was applied to determine the antibiotic susceptibility of $R$. anatipestifer isolates. Twenty-one antibiotic discs (Oxoid, UK) were used, including: $30 \mu \mathrm{g}$ of amikacin (AK30), $30 \mu \mathrm{g}$ of amoxicillin/clavulanic acid (AMC30), $10 \mu \mathrm{g}$ of ampicillin (AM10), $10 \mu \mathrm{g}$ of bacitracin (B10), $30 \mu \mathrm{g}$ of cephalothin (KF30), $30 \mu \mathrm{g}$ of cefuroxime sodium (CXM30), $30 \mu \mathrm{g}$ of cefotaxime (CTX30), $5 \mu \mathrm{g}$ of ciprofloxacin (CIP5), $2 \mu \mathrm{g}$ of clindamycin (CC2), $10 \mu \mathrm{g}$ of colistin (CL10), $15 \mu \mathrm{g}$ of erythromycin (E15), $10 \mu \mathrm{g}$ of gentamicin (GM10), $2 \mu \mathrm{g}$ of lincomycin (MY2), $30 \mu \mathrm{g}$ of nalidixic acid (NA30), $30 \mu \mathrm{g}$ of neomycin (N30), $10 \mu \mathrm{g}$ of norfloxacin (NOR10), $5 \mu \mathrm{g}$ of ofloxacin (OFX5), 10U of penicillin $\mathrm{G}$ (P10), $10 \mu \mathrm{g}$ of streptomycin (S10), 23.75/1.25 $\mu \mathrm{g}$ of sulphamethoxazole/trimethoprim (SXT25), and $30 \mu \mathrm{g}$ of tetracycline (TE30). Among these antibiotics, amoxicillin/clavulanic acid, ampicillin, colistin, erythromycin, gentamicin, lincomycin, nalidixic acid, neomycin, penicillin, streptomycin, sulphamethoxazole, trimethoprim, and tetracycline are approved for use in waterfowl in Taiwan (19). A 0.5 McFarland bacterial suspension in sterile normal saline was inoculated on Mueller Hinton Agar supplemented with 5\% sheep blood (Becton Dickinson, USA), and the antibiotic discs were placed on the agar plates, which were then 
cultured for $24 \mathrm{~h}$ at $37^{\circ} \mathrm{C}$ with $5 \% \mathrm{CO}_{2}$. The inhibition zone diameters were interpreted according to the interpretive criteria in the Clinical and Laboratory Standards Institute (USA) guidelines (33).

\section{Results}

The results of a pilot survey for studying the prevalence of $R$. anatipestifer in fowls living in slaughterhouses demonstrated that 375 duck samples in 15 flocks and the same number of goose samples and flocks are required for an accepted error of $10 \%$ and a confidence level of $95 \%$. In this study, 25 swabs for each of duck and goose flocks were collected in three waterfowl slaughterhouses in central Taiwan between 2009 and 2010. The flock prevalences of $R$. anatipestifer in living ducks and geese in slaughterhouses were $60.0 \%(9 / 15)$ and $53.3 \%(8 / 15)$, while the individual prevalences in ducks and geese were $12.3 \%(46 / 375)$ and $8.0 \%$ (30/375), respectively. Almost all $(97.4 \%, 74 / 76) R$. anatipestifer isolates were cultured from laryngeal specimens, including 44 from ducks and 30 from geese, whereas two were isolated from conjunctivas of ducks (Table 1). Seasonal distribution of $R$. anatipestifer in fowls in slaughterhouses showed the highest incidence in spring (69.6\% in ducks, $36.7 \%$ in geese, subtotal $56.6 \%$ ) and the lowest in autumn ( $0 \%$ in ducks, $16.7 \%$ in geese, subtotal $6.6 \%$ ) (Table 2).

To analyse the presence of $R$. anatipestifer in fowl during the slaughtering process, 125 ducks collected from 5 equal groups were examined by both bacterial isolation and PCR detection. The isolation rates for birds on arrival, birds in holding, and birds in the defeathering and degumming stages were $65.6 \%$, $76.0 \%, 1.6 \%$, and $0 \%$, respectively; and PCR detection rates were correspondingly $65.6 \%, 76.0 \%, 21.6 \%$, and $11.2 \%$. No discrepancies were detected in living fowls; however, a rate discrepancy between bacterial isolation and PCR detection was found in carcass samples (Table 3).

Additionally, an environmental survey of $R$. anatipestifer in waterfowl slaughterhouses was also conducted. Various samples including pool water, bottom mud, faeces, drinking water, and fodder were inspected by both isolation and PCR examination. No $R$. anatipestifer isolates were found in these samples, but nearly all kinds of samples were contaminated with the DNA of $R$. anatipestifer, with especially high detection rates in the samples from pool water $(57.0 \%)$, bottom mud $(83.0 \%)$, and faeces $(40.0 \%)$ (Table 4$)$. Two swabs of the surfaces of workbenches grew $R$. anatipestifer colonies but no swabs from the various processing machines (defeathering machine, scalding machine, and transport machine) grew any. Thereafter, extracted DNA from swabs on the surfaces of the workbench (7.0\%), defeathering machines (30.0\%), scalding machines $(10.0 \%)$, but not transport machines, gave positive results for the presence of $R$. anatipestifer (Table 5). Meanwhile, borehole water and various types of wastewater samples were inspected using both bacterial culture and PCR detection. These samples formed no colonies, but the DNA collected from them regarding waste feathers $(26.7 \%)$, defeathering waste $(10.0 \%)$, and slaughter waste $(10.0 \%)$ showed positive rates (Table 6).

Serotype characterisation by agglutination was performed on all $R$. anatipestifer isolates. The distribution of serotypes in the slaughterhouse isolates showed that serotype B was the dominant serotype in both duck (34.8\%) and geese (46.7\%) samples, followed by serotypes $3(30.4 \%)$ and $21(28.3 \%)$ in ducks, and serotypes $21(40.0 \%)$ and $2(23.3 \%)$ in geese (Table 7). None of the serotypes 7, 12, 13, 16, or 18 were found in the isolates in this study. Twenty-one common antibiotics were used to evaluate the antibiotic susceptibility of the slaughterhouse isolates. Twelve showed $50 \%$ or higher efficacies for the duck isolates, including amoxicillin/clavulanic acid (98\%), cefotaxime (96\%), cefuroxime sodium (93\%), ofloxacin (83\%), norfloxacin $(80 \%)$, cephalothin $(78 \%)$, ciprofloxacin (78\%), tetracycline (65\%), penicillin G (57\%), clindamycin (52\%), erythromycin (52\%), and ampicillin (50\%); while for the geese isolates, the 10 antimicrobials amoxicillin/clavulanic acid (97\%), cefuroxime sodium (90\%), cefotaxime (87\%), cephalothin $(80 \%)$, ofloxacin $(77 \%)$, ampicillin $(60 \%)$, sulphamethoxazole/ trimethoprim (57\%), ciprofloxacin (53\%), norfloxacin (53\%), and penicillin G $(50 \%)$ had good efficacies. Most importantly, $50 \%$ or more of the isolates had developed resistance against several antibiotics, such as colistin (100\%), lincomycin (76\%), nalidixic acid $(72 \%)$, neomycin $(70 \%)$, gentamicin (67\%), and sulphamethoxazole/trimethoprim (50\%) in duck isolates, or colistin (100\%), lincomycin (83\%), nalidixic acid (63\%), neomycin (63\%), streptomycin (57\%), and gentamicin (50\%) in geese isolates (Table 8). Notably, all the slaughterhouse isolates were resistant to colistin.

Table 1. The prevalence of Riemerella anatipestifer in living waterfowl in Taiwan slaughterhouses

\begin{tabular}{llll}
\hline Specimen source & \multicolumn{3}{c}{ Number of positive samples $(\%)$} \\
\cline { 2 - 4 } & Duck $(\mathrm{n}=375)$ & Goose $(\mathrm{n}=375)$ & Total $(\mathrm{n}=750)$ \\
\hline Larynx & $44(11.7)^{*}$ & $30(8.0)$ & $74(9.9)$ \\
Conjunctiva & $2(0.5)^{*}$ & $0(0)$ & $2(0.3)$ \\
Total & $46(12.3)$ & $30(8.0)$ & $76(10.1)$ \\
\hline
\end{tabular}

$* \mathrm{p}<0.05$ 
Table 2. The prevalence of Riemerella anatipestifer in waterfowl in different seasons

\begin{tabular}{lllll}
\hline \multirow{2}{*}{ Waterfowl } & \multicolumn{4}{c}{ Number of positive samples (\%) } \\
\cline { 2 - 5 } & Spring & Summer & Autumn & Winter \\
\hline Duck $(\mathrm{n}=46)$ & $32(69.6)^{*}$ & $5(10.9)^{*}$ & $0(0)$ & $9(19.6)^{*}$ \\
Goose $(\mathrm{n}=30)$ & $11(36.7)$ & $6(20.0)$ & $5(16.7)$ & $8(26.7)$ \\
Prevalence & $43(56.6)$ & $11(14.5)$ & $5(6.6)$ & $17(22.4)$ \\
\hline
\end{tabular}

$* \mathrm{p}<0.05$

Table 3. The detection rates of Riemerella anatipestifer in waterfowl in various stages in Taiwan slaughterhouses

\begin{tabular}{lllll}
\hline \multirow{2}{*}{ Flock } & \multicolumn{4}{c}{ Number of positive detections $(\%)(\mathrm{n}=25)$} \\
\cline { 2 - 5 } & On arriving & Holding & Defeathered & Degummed \\
\hline 1 & $18(72.0)$ & $11(44.0)$ & $11(44.0)$ & $5(20.0)$ \\
2 & $6(24.0)$ & $22(88.0)$ & $5(20.0)$ & $3(12.0)$ \\
3 & $25(100)$ & $21(84.0)$ & $5(20.0)$ & $0(0.0)$ \\
4 & $21(84.0)$ & $22(88.0)$ & $4(16.0)$ & $4(16.0)$ \\
5 & $12(48.0)$ & $19(76.0)$ & $4(16.0)$ & $2(8.0)$ \\
\hline PCR & $82(65.6)$ & $95(76.0)$ & $27(21.6)$ & $14(11.2)$ \\
\hline Isolation & $82(65.6)$ & $95(76.0)$ & $2(1.6)$ & $0(0)$ \\
\hline
\end{tabular}

Table 4. The detection rates of Riemerella anatipestifer in environmental samples from holding areas in Taiwan slaughterhouses

\begin{tabular}{|c|c|c|c|c|c|}
\hline \multirow{2}{*}{$\begin{array}{l}\text { Sampling } \\
\text { scheme }\end{array}$} & \multicolumn{5}{|c|}{ Number of positive detections $(\%)(n=10)$} \\
\hline & Pool water & Bottom mud & Faeces & Drinking water & Fodder \\
\hline 1 & $7(70.0)$ & $9(90.0)$ & $5(50.0)$ & $0(0)$ & $0(0)$ \\
\hline 2 & $5(50.0)$ & $8(80.0)$ & $3(30.0)$ & $1(10.0)$ & $0(0)$ \\
\hline 3 & $5(50.0)$ & $8(80.0)$ & $4(40.0)$ & $2(20.0)$ & $0(0)$ \\
\hline PCR & $17(57.0)$ & $25(83.0)$ & $12(40.0)$ & $3(10.0)$ & $0(0)$ \\
\hline Isolation & $0(0)$ & $0(0)$ & $0(0)$ & $0(0)$ & $0(0)$ \\
\hline
\end{tabular}

Table 5. The detection rates of Riemerella anatipestifer in samples from workspace in Taiwan slaughterhouses

\begin{tabular}{lllll}
\hline \multirow{2}{*}{ Sampling scheme } & \multicolumn{4}{c}{ Number of positive detections $(\%)(\mathrm{n}=10)$} \\
\cline { 2 - 5 } & Workbench top & Defeather machine & Scalding machine & Transport machine \\
\hline 1 & $2(20.0)$ & $4(40.0)$ & $2(20.0)$ & $0(0)$ \\
2 & $0(0)$ & $3(30.0)$ & $0(0)$ & $0(0)$ \\
3 & $0(0)$ & $2(20.0)$ & $1(10.0)$ & $0(0)$ \\
\hline PCR & $2(7.0)$ & $9(30.0)$ & $3(10.0)$ & $0(0)$ \\
\hline Isolation & $2(7.0)$ & $0(0)$ & $0(0)$ & $0(0)$ \\
\hline
\end{tabular}

Table 6. The detection rates of Riemerella anatipestifer in water samples in Taiwan slaughterhouses

\begin{tabular}{|c|c|c|c|c|c|c|c|}
\hline \multirow{3}{*}{$\begin{array}{l}\text { Sampling } \\
\text { scheme }\end{array}$} & \multicolumn{7}{|c|}{ Number of positive detections $(\%)(n=10)$} \\
\hline & \multirow{2}{*}{$\begin{array}{l}\text { Water } \\
\text { supply }\end{array}$} & \multirow{2}{*}{$\begin{array}{l}\text { Waste } \\
\text { feather }\end{array}$} & \multicolumn{5}{|c|}{$\begin{array}{ll}\text { Waste water } \\
\end{array}$} \\
\hline & & & Scalding & Defeather & Degumming & Organ purge & Slaughter \\
\hline 1 & $0(0)$ & $1(10.0)$ & $0(0)$ & $1(10.0)$ & $0(0)$ & $0(0)$ & $0(0)$ \\
\hline 2 & $0(0)$ & $3(30.0)$ & $0(0)$ & $0(0)$ & $0(0)$ & $0(0)$ & $2(20.0)$ \\
\hline 3 & $0(0)$ & $4(40.0)$ & $0(0)$ & $2(20.0)$ & $0(0)$ & $0(0)$ & $1(10.0)$ \\
\hline PCR & $0(0)$ & $8(26.7)$ & $0(0)$ & $3(10.0)$ & $0(0)$ & $0(0)$ & $3(10.0)$ \\
\hline Isolation & $0(0)$ & $0(0)$ & $0(0)$ & $0(0)$ & $0(0)$ & $0(0)$ & $0(0)$ \\
\hline
\end{tabular}


Table 7. The serotype of Riemerella anatipestifer isolated from waterfowl slaughterhouses

\begin{tabular}{|c|c|c|c|}
\hline \multirow{2}{*}{ Serotype } & \multicolumn{3}{|c|}{ Isolates (\%) } \\
\hline & Ducks $(n=46)$ & Geese $(n=30)$ & Total \\
\hline 1 & $1(2.2)$ & $1(3.3)$ & $2(2.6)$ \\
\hline 2 & $0(0)$ & $7(23.3)$ & $7(9.2)$ \\
\hline 3 & $14(30.4)$ & $0(0)$ & $14(18.4)$ \\
\hline 4 & $2(4.3)$ & $3(10.0)$ & $5(6.6)$ \\
\hline 5 & $1(2.2)$ & $0(0)$ & $1(1.3)$ \\
\hline 6 & $7(15.2)$ & $1(3.3)$ & $8(10.5)$ \\
\hline 7 & $0(0)$ & $0(0)$ & $0(0)$ \\
\hline 8 & $8(17.4)$ & $2(6.7)$ & $10(13.2)$ \\
\hline 9 & $4(8.7)$ & $5(16.7)$ & $9(11.8)$ \\
\hline 10 & $1(2.2)$ & $0(0)$ & $1(1.3)$ \\
\hline 11 & $9(19.6)$ & $0(0)$ & $9(11.8)$ \\
\hline 12 & $0(0)$ & $0(0)$ & $0(0)$ \\
\hline 13 & $0(0)$ & $0(0)$ & $0(0)$ \\
\hline 14 & $4(8.7)$ & $3(10.0)$ & $7(9.2)$ \\
\hline 15 & $4(8.7)$ & $0(0)$ & $4(5.3)$ \\
\hline 16 & $0(0)$ & $0(0)$ & $0(0)$ \\
\hline 17 & $1(2.2)$ & $0(0)$ & $1(1.3)$ \\
\hline 18 & $0(0)$ & $0(0)$ & $0(0)$ \\
\hline 19 & $1(2.2)$ & $0(0)$ & $1(1.3)$ \\
\hline 20 & $1(2.2)$ & $5(16.7)$ & $6(7.9)$ \\
\hline 21 & $13(28.3)$ & $12(40.0)$ & $25(32.9)$ \\
\hline B & $16(34.8)$ & $14(46.7)$ & $30(39.5)$ \\
\hline Unknown & $8(17.4)$ & $3(10.0)$ & $11(14.5)$ \\
\hline
\end{tabular}

Table 8. The antibiotic susceptibility of Riemerella anatipestifer isolated from waterfowl slaughterhouses (46 ducks, 30 geese)

\begin{tabular}{|c|c|c|c|c|c|c|c|c|c|}
\hline \multirow{3}{*}{ Antibiotic } & \multicolumn{9}{|c|}{ Number of positive isolates (\%) } \\
\hline & \multicolumn{3}{|c|}{ Resistance } & \multicolumn{3}{|c|}{ Intermediate } & \multicolumn{3}{|c|}{ Susceptibility } \\
\hline & Duck & Goose & Subtotal & Duck & Goose & Subtotal & Duck & Goose & Subtotal \\
\hline Amikacin (AK30) & $35(76.0)$ & $20(67.0)$ & $55(72.4)$ & $1(2.2)$ & $5(16.7)$ & $6(7.9)$ & $10(21.2$ & $5(16.7)$ & $15(19.7)$ \\
\hline Amoxicillin/Clavulanic acid (AMC30) & $1(2.2)$ & $1(3.3)$ & $2(2.6)$ & $0(0)$ & $0(0)$ & $0(0)$ & $45(97.8)$ & $29(96.7)$ & $74(97.4)$ \\
\hline Ampicillin (AM10) & $19(41.3)$ & $7(23.3)$ & $26(34.2)$ & $4(8.7)$ & $5(16.7)$ & $9(11.8)$ & $23(50.0)$ & $18(60.0)$ & $41(53.9)$ \\
\hline Bacitracin (B10) & $6(13.0)$ & $11(36.7)$ & $17(22.4)$ & $19(41.3)$ & $5(16.7)$ & $24(31.6)$ & $21(45.7)$ & $14(46.7)$ & $35(46.1)$ \\
\hline Cephalothin (KF30) & $2(4.3)$ & $4(13.3)$ & $6(7.9)$ & $8(17.4)$ & $2(6.7)$ & $10(13.2)$ & $36(78.3)$ & $24(80.0)$ & $60(78.9)$ \\
\hline Cefuroxime Sodium (CXM30) & $1(2.2)$ & $2(6.7)$ & $3(3.9)$ & $2(4.3)$ & $1(3.3)$ & $3(3.9)$ & $43(93.5)$ & $27(90.0)$ & $70(92.1)$ \\
\hline Cefotaxime (CTX30) & $1(2.2)$ & $4(13.3)$ & $5(6.6)$ & $1(2.2)$ & $0(0)$ & $1(1.3)$ & $44(95.7)$ & $26(86.7)$ & $70(92.1)$ \\
\hline Ciprofloxacin (CIP5) & $6(13.0)$ & $7(23.3)$ & $13(17.1)$ & $4(8.7)$ & $7(23.3)$ & $11(14.5)$ & $36(78.3)$ & $16(53.3)$ & $52(68.4)$ \\
\hline Clindamycin (CC2) & $19(41.3)$ & $13(43.3)$ & $32(42.1)$ & $3(6.5)$ & $3(10.0)$ & $6(7.9)$ & $24(52.2)$ & $14(46.7)$ & $38(50)$ \\
\hline Colistin (CL10) & $46(100)$ & $30(100)$ & $76(100)$ & $0(0)$ & $0(0)$ & $0(0)$ & $0(0)$ & $0(0)$ & $0(0)$ \\
\hline Erythromycin (E15) & $12(26.1)$ & $12(40.0)$ & $24(31.6)$ & $10(21.2)$ & $6(20.0)$ & $16(21.1)$ & $24(52.2)$ & $12(40.0)$ & $36(47.4)$ \\
\hline Gentamicin (GM10) & $31(67.4)$ & $15(50.0)$ & $46(60.5)$ & $2(4.3)$ & $6(20.0)$ & $8(10.5)$ & $13(28.3)$ & $9(30.0)$ & $22(28.9)$ \\
\hline Lincomycin (MY2) & $35(76.1)$ & $25(83.3)$ & $60(78.9)$ & $9(19.6)$ & $5(16.7)$ & $14(18.4)$ & $2(4.3)$ & $0(0)$ & $2(2.6)$ \\
\hline Nalidixic acid (NA30) & $33(71.7)$ & $19(63.3)$ & $52(68.4)$ & $5(10.9)$ & $7(23.3)$ & $12(15.8)$ & $8(17.4)$ & $4(13.3)$ & $12(15.8)$ \\
\hline Neomycin (N30) & $32(69.6)$ & $19(63.3)$ & $51(67.1)$ & $7(15.2)$ & $11(36.7)$ & $18(23.7)$ & $7(15.2)$ & $0(0)$ & $7(9.2)$ \\
\hline Norfloxacin (NOR10) & $5(10.9)$ & $11(36.7)$ & $16(21.1)$ & $4(8.7)$ & $3(10.0)$ & $7(9.2)$ & $37(80.4)$ & $16(53.3)$ & $53(69.7)$ \\
\hline Ofloxacin (OFX5) & $3(6.5)$ & $3(10.0)$ & $6(7.9)$ & $5(10.9)$ & $4(13.3)$ & $9(11.8)$ & $38(82.6)$ & $23(76.7)$ & $61(80.3)$ \\
\hline Penicillin G (P10) & $5(10.9)$ & $9(30.0)$ & $14(18.4)$ & $15(32.6)$ & $6(20.0)$ & $21(27.6)$ & $26(56.5)$ & $15(50.0)$ & $41(53.9)$ \\
\hline Streptomycin (S10) & $29(63.0)$ & $17(56.7)$ & $46(60.5)$ & $5(10.9)$ & $5(16.7)$ & $10(13.2)$ & $12(26.1)$ & $8(26.7)$ & $20(26.3)$ \\
\hline $\begin{array}{l}\text { Sulphamethoxazole/ } \\
\text { Trimethoprim (SXT25) }\end{array}$ & $23(50.0)$ & $5(16.7)$ & $28(36.8)$ & $4(8.7)$ & $8(26.7)$ & $12(15.8)$ & $19(41.3)$ & $17(56.7)$ & $36(47.4)$ \\
\hline Tetracycline (TE30) & $7(15.0)$ & $10(33.3)$ & $17(22.4)$ & $9(19.6)$ & $9(30.0)$ & $18(23.7)$ & $30(65.2)$ & $11(36.7)$ & $41(53.9)$ \\
\hline
\end{tabular}




\section{Discussion}

Although $R$. anatipestifer infection is nonlethal for adult waterfowl, there is an economic rationale for preventing infestations of the bacterium. The microbe's biofilm formation and antibiotic resistance nevertheless makes the eradication of its contamination in the field nearly impossible $(13,29)$. The insufficient biosecurity and inadequate husbandry methods of waterfowl farms and slaughterhouses increase the exposure of waterfowl to $R$. anatipestifer and even favour its silent circulation in fields, thereby leading to possible outbreaks without effective treatment. This study is the first survey of $R$. anatipestifer in waterfowl slaughterhouses in Taiwan. A few of the healthy ducks and geese that arrived at the slaughterhouses proved to be infected with $R$. anatipestifer (Table 1). Ducks appear to be more susceptible to $R$. anatipestifer than geese, although they are closely related, belonging to the same Anatidae family. It was reported that outbreaks of $R$. anatipestifer infection usually occur in spring and winter or after bad weather with a sudden temperature drop $(9,30)$; this was confirmed by the high incidences observed between November and April in this study (Table 2). Interestingly, most of the isolates (97.4\%) were cultured from laryngeal samples of healthy waterfowl. The result was in agreement with previous suggestions that $R$. anatipestifer is a normal part of the pharyngeal flora of some ducks $(2,27)$. It is worth noting that the comparatively easy method of conjunctiva sampling, which is used to isolate pathogens from sick fowl and may appear to offer a simpler alternative to laryngeal sampling, is not suited for general surveys of $R$. anatipestifer. In agreement with a previous study on $R$. anatipestifer infection in wild birds (2), we suggest that laryngeal swabs are most suitable to isolate the bacteria.

The prevalence of $R$. anatipestifer in ducks being held in a slaughterhouse was higher than that of ducks on arrival, which suggests that some ducks were infected in a contaminated environment during the holding period or possibly that the stress of transportation and crowding increased the detection rate of $R$. anatipestifer in the laryngeal bacteria of healthy ducks. The high-temperature treatments of scalding $\left(64^{\circ} \mathrm{C}\right.$ for $\left.3 \mathrm{~min}\right)$ and rosin plucking $\left(120-150^{\circ} \mathrm{C}\right.$ for $\left.5 \mathrm{~s}\right)$ are detrimental to the survival of $R$. anatipestifer, therefore only $1.6 \%$ of defeathered ducks and no degummed duck carcasses were positively isolated; however, a few carcass washing samples yielded bacteria to positive detection through the PCR method due to the remaining nucleic acids (Table 3). In the environmental survey of $R$. anatipestifer in slaughterhouses, only two isolates were cultured from workbench swabs, but none were isolated from any waste or swab samples. However, most of the environmental samples showed a low positive rate through PCR detection, confirming that any $R$. anatipestifer surviving in contaminated environments may be too fragile to be cultivated (10). Therefore, the bacterial culture method may not truly reflect the level of $R$. anatipestifer contamination in waterfowl farms.

The serotypes of the slaughterhouse $R$. anatipestifer isolates revealed that type B was the major serotype in both ducks $(34.8 \%)$ and geese $(46.7 \%)$. Serotype B, which does not belong to the classic 21 serotypes (25), was discovered, classified, and reported as one of the dominant serotypes (serotypes 2, 1, and B) in sick waterfowl in a farm survey study conducted in 2010 (6). Sixteen serotypes were identified in duck isolates, while ten serotypes were found in geese isolates in this study. This suggests that ducks are susceptible to more serotypes of $R$. anatipestifer than geese. Interestingly, multiple serotypes were identified in one waterfowl, which clearly proved that broad cross-protection is not established in $R$. anatipestifer infections. As several serotypes were found circulating in the same slaughterhouses, the development of an effective vaccine may be difficult.

According to the results of disc diffusion assays, 21 antibiograms of the isolates showed that all $R$. anatipestifer isolates are resistant to colistin and more than $70 \%$ of the isolates are resistant to lincomycin. These antibiotics were approved for use in feed and for treating bacterial infection in poultry, as well as neomycin $(70.0 \%$ and $63.0 \%$ resistance in duck and geese isolates, respectively). Interestingly, the bacterium did not demonstrate elevated resistance rates to penicillin $\mathrm{G}$, also an approved feed drug. More than half of the isolates had developed resistance against amikacin, gentamicin, nalidixic acid, and streptomycin, which are not allowed for use in feed or environmental treatments. It seems that overuse or misuse of approved antibiotics in food animals is a major cause of the antibiotic resistance problem but not the only one. Moreover, multiple drug resistances were found in most isolates. Antibiotic resistance makes the treatment and control of $R$. anatipestifer difficult in the waterfowl industry. Fortunately, a high percentage $(97.4 \%)$ of the tested isolates were still susceptible to amoxicillin, which is approved for use in waterfowl.

This study demonstrated that $R$. anatipestifer has substantially contaminated waterfowl slaughterhouses and circulates in waterfowl farms and slaughterhouses in central Taiwan. The high prevalence of colistin resistance in the isolates associates with a risk of dissemination of that resistance gene in agricultural soils, probably through a plasmid-mediated route (21). Therefore, antibiotic therapy action might be suppressed in a zoonotic infection with the bacteria which have acquired the multidrug resistance. It is worth noting that the bacterial isolation method may not suit $R$. anatipestifer surveys on farms. There is a salient and urgent need for training in farm biosecurity with a particular focus on $R$. anatipestifer in 
the waterfowl industry. Meanwhile, the severe problem of antibiotic resistance in $R$. anatipestifer requires the attention of the appropriate antimicrobial stewards.

Conflict of Interests Statement: The authors declare that there is no conflict of interests regarding the publication of this article.

Financial Disclosure Statement: This work was supported in part by a grant from the Ministry of Science and Technology (MOST 104-2320-B-415-005) and a grant from the Department of Industrial Technology, Ministry of Economic Affairs (MOEA105-EC-17-A-21-0337) of Taiwan.

Animal Rights Statement: The authors declare that the experiments on animals were conducted in accordance with local Ethical Committee laws and regulations as regards the care and use of laboratory animals.

\section{References}

1. Brogden K.A., Rhoades K.R., Rimler R.B.: Serologic types and physiologic characteristics of 46 avian Pasteurella anatipestifer cultures. Avian Dis 1982, 26, 891-896.

2. Cha S.Y., Seo H.S., Wei B., Kang M., Roh J.H., Yoon R.H., Kim J.H., Jang H.K.: Surveillance and characterization of Riemerella anatipestifer from wild birds in South Korea. J Wildl Dis 2015, 51, 341-347.

3. Chang C.F.: Pathogenesis of Pasteurella anatipestifer infection in ducks and drug sensitivity. Taiwan J Vet Med Anim Hus 1984, 43, 40-46.

4. Chen C.L., Wang S.T., Chu C., Wang S.H.: Comparison of four molecular typing methods for Riemerella anatipestifer. Taiwan Vet J 2015, 41, 177-185.

5. Chen Y.P., Lee S.H., Chou C.H., Tsai H.J.: Detection of florfenicol resistance genes in Riemerella anatipestifer isolated from ducks and geese. Vet Microbiol 2012, 154, 325-331.

6. Chen Y.P., Lee S.H., Tsai H.J.: Serotyping of Riemerellar anatipestifer isolates from waterfowl in Taiwan between 2008 and 2012. Exp Rep Taiwan AHRI 2013, 48, 21-28.

7. Chu C.Y., Liu C.H., Liou J.J., Lee J.W., Cheng L.T.: Development of a subunit vaccine containing recombinant Riemerella anatipestifer outer membrane protein $\mathrm{A}$ and $\mathrm{CpG}$ ODN adjuvant. Vaccine 2015, 33, 92-99.

8. Cochran W.G.: Sampling Techniques, Wiley, New York. 1977.

9. Cooper G.L.: Pasteurella anatipestifer infections in California turkey flocks: circumstantial evidence of a mosquito vector. Avian Dis 1989, 33, 809-815.

10. Davis M.A., Winn J.F.: Poultry disease manual. Texas Cooperative Extension, Texas A \& M University System, College Station, Texas. 2006.

11. Heddleston K.L.: Infectious serositis. In: Diseases of poultry. Edited by Hofstad M.S., Calnek B.W., Biester H.E. American Association of Avian Pathologists, Iowa State University Press, Ames 1972, pp. 246-251.

12. Hess C., Enichlmayr H., Jandreski-Cvetkovic D., Liebhart D., Bilic, I., Hess M.: Riemerella anatipestifer outbreaks in commercial goose flocks and identification of isolates by MALDI-TOF mass spectrometry. Avian Pathol 2013, 42, $151-156$.

13. Hu Q., Han X., Zhou X., Ding S., Ding C., Yu S.: Characterization of biofilm formation by Riemerella anatipestifer. Vet Microbiol 2010, 144, 429-436.
14. Huang C.C.: The natural transmission, serotypes, antimicrobial susceptibility of Riemerella anatipestifer and combinational infection with circoviruses in waterfowl. Department of Veterinary Medicine. National Chiayi University, Chiayi, Taiwan. 2008

15. Layton H.W., Sandhu T.S.: Protection of ducklings with a brothgrown Pasteurella anatipestifer bacterin. Avian Dis 1984, 28, 718-726.

16. Leavitt S., Ayroud M.: Riemerella anatipestifer infection of domestic ducklings. Can Vet J 1997, 38, 113.

17. Leibovitz L.: A survey of the so-called "anatipestifer syndrome". Avian Dis 1972, 16, 836-851.

18. Li Y.L.: Sequence analysis of plasmids and application to detection of antibiotics resistance genes in Riemerella anatipestifer isolated in Taiwan. Department of Veterinary Medicine. National Chung Hsing University, Taichung. 2004. p. 75 .

19. Liu C.K., Chen C.M., Chang T.C., Chang W.F., Chang S.K., Kuo C.C., Lin J.S., Chu C.Y.: Handbook of veterinary drugs commonly used for poultry. Bureau of Animal and Plant Health Inspection and Quarantine, COA, EY, Taipei, Taiwan, R.O.C. 2009.

20. Liu H., Wang X., Ding C., Han X., Cheng A., Wang S., Yu S.: Development and evaluation of a trivalent Riemerella anatipestifer-inactivated vaccine. Clin Vaccine Immunol 2013, 20, 691-697.

21. Liu Y.Y., Wang Y., Walsh T.R., Yi L.X., Zhang R., Spencer J., Doi Y., Tian G., Dong B., Huang X., Yu L.F., Gu D., Ren H., Chen X., Lv L., He D., Zhou H., Liang Z., Liu J.H., Shen J.: Emergence of plasmid-mediated colistin resistance mechanism MCR-1 in animals and human beings in China: a microbiological and molecular biological study. Lancet Infect Dis 2016, 16, 161-168.

22. Loh H., Teo T.P., Tan H.C.: Serotypes of 'Pasteurella' anatipestifer isolates from ducks in Singapore: a proposal of new serotypes. Avian Pathol 1992, 21, 453-459.

23. Magwedere K., Rauff D., De Klerk G., Keddy K.H., Dziva F.: Incidence of nontyphoidal Salmonella in food-producing animals, animal feed, and the associated environment in South Africa, 2012-2014. Clin Infect Dis 2015, 61, 283-289.

24. Pathanasophon P., Sawada T., Pramoolsinsap T., Tanticharoenyos T.: Immunogenicity of Riemerella anatipestifer broth culture bacterin and cell-free culture filtrate in ducks. Avian Pathol 1996, 25, 705-719.

25. Pathanasophon P., Sawada T., Tanticharoenyos T.: New serotypes of Riemerella anatipestifer isolated from ducks in Thailand. Avian Pathol 1995, 24, 195-199.

26. Phonvisay M., Liou J.J., Cheng L.T., Chen Y.P., Wu H.C., Liu C.H., Lee J.W., Chu C.Y.: Survey of a Riemerella anatipestifer outbreak in southern taiwan duck farms. Taiwan Vet J 2017, 43, 165-170.

27. Ryll M., Christensen H., Bisgaard M., Christensen J.P., Hinz K.H., Köhler B.: Studies on the prevalence of Riemerella anatipestifer in the upper respiratory tract of clinically healthy ducklings and characterization of untypable strains. J Vet Med B Infect Dis Vet Public Health 2001, 48, 537-546.

28. Sandhu T.S.: Immunogenicity and safety of a live Pasteurella anatipestifer vaccine in White Pekin ducklings: laboratory and field trials. Avian Pathol 1991, 20, 423-432.

29. Sandhu T.S.: Riemerella anatipestifer infection. In: Diseases of Poultry, edited by Saif Y.M., Barnes H.J., Glisson J.R., Fadly A.M., McDougald L.R., Swayne D.E., Iowa State Press, Ames, Iowa. 2003. pp. 676-682.

30. Tian K.G., Gui Y.P.: Research on infectious sinusitis in white Beijing ducklings. I. Epidemiology and pathology. Xu Mu Shou Yi Xue Bao 1990, 21, 327-331.

31. Tsai H.J., Liu Y.T., Tseng C.S., Pan M.J.: Genetic variation of the ompA and 16S rRNA genes of Riemerella anatipestifer. Avian Pathol 2005, 34, 55-64.

32. Umaraw P., Prajapati A., Verma A.K., Pathak V., Singh V.P.: Control of campylobacter in poultry industry from farm to 
poultry processing unit: A review. Crit Rev Food Sci Nutr 2017, 57, 659-665.

33. Wayne P.: CLSI. Performance Standards for Antimicrobial Susceptibility Testing; Twenty-Fifth Informational Supplement. CLSI Document M100-S25, Clinical and Laboratory Standards Institute. 2015.
34. Yu C.Y., Liu Y.W., Chou S.J., Chao M.R., Weng B.C., Tsay J.G., Chiu C.H., Ching Wu C., Long Lin T., Chang C.C., Chu C.: Genomic diversity and molecular differentiation of Riemerella anatipestifer associated with eight outbreaks in five farms. Avian Pathol 2008, 37, 273-279. 